Itinéraires Itinéraires

Littérature, textes, cultures

\title{
Dans la jungle de l'intime : enquête lexicographique et lexicométrique (1606-2008)
}

\section{Véronique Montémont}

\section{OpenEdition}

\section{Journals}

Édition électronique

URL : http://journals.openedition.org/itineraires/585

DOI : 10.4000/itineraires.585

ISSN : 2427-920X

Éditeur

Pléiade

\section{Édition imprimée}

Date de publication : 1 décembre 2009

Pagination : 15-38

ISBN : 978-2-296-10791-5

ISSN : 2100-1340

\section{Référence électronique}

Véronique Montémont, «Dans la jungle de l'intime : enquête lexicographique et lexicométrique (1606-2008) », Itinéraires [En ligne], 2009-4 | 2009, mis en ligne le 04 septembre 2014, consulté le 19 avril 2019. URL : http://journals.openedition.org/itineraires/585; DOI : 10.4000/itineraires.585

Ce document a été généré automatiquement le 19 avril 2019

\section{(c)}

Itinéraires est mis à disposition selon les termes de la licence Creative Commons Attribution - Pas d'Utilisation Commerciale - Pas de Modification 4.0 International. 


\title{
Dans la jungle de l'intime : enquête lexicographique et lexicométrique (1606-2008)
}

\author{
Véronique Montémont
}

1 L'intime est aujourd'hui au centre d'un étrange paradoxe, régulièrement souligné par ses théoriciens, et que résume fort bien la formule d'Élisabeth Lebovici : désormais l'intimité est « enfouie et fouillée, dedans et dehors ${ }^{1}$ ». La notion renvoie, étymologiquement (et de surcroît, sous une forme superlative) à la dimension la plus intérieure de l'expérience humaine, et dans une certaine mesure, à la moins communicable, à cause de son caractère foncièrement privé. Au reste, il n'est pas rare que l'intime, dans la tradition littéraire moderne, soit associé au « secret ", terme avec lequel on peut trouver de nombreuses cooccurrences littéraires: aussi bien dans l'œuvre de Maine de Biran (qui parle de « rapports secrets et intimes » entre le corps et l'âme ${ }^{2}$ ), que dans celle de Nathalie Sarraute (« ces grandes pages gondolées [...] d'où émane quelque chose d'intime, de secret $\left.{ }^{3} \gg\right)$, en passant par celles de Maurice de Guérin, Jules Romains ou Simone de Beauvoir. Mais dans le même temps, le mot est survalorisé dans la sphère publique, où le $\mathrm{xx}^{\mathrm{e}}$ siècle lui réserve une fortune exponentielle : qualifier des écrits, un récit, voire des interviews télévisées d'intimes revient à leur conférer une estampille singulière, la valeur ajoutée de l'authenticité, de la vérité, de l'émotion, dont il semble désormais aller de soi qu'elle puisse être partagée avec des milliers d'autres individus. C'est ainsi que le numéro 1644 de VSD de février 2009 nous proposait en couverture un " Yannick Noah intime », livrant « ce qu'[il] n'avait jamais dit » dans une « biographie sans tabou ».

2 Plusieurs auteurs soulignent cette ambiguïté constitutive de la notion d'intime, cette tension entre public et privé, que l'évolution des codes sociaux et des pratiques culturelles a rendue inévitable. Ainsi Jean-Pierre Dufief soulève-t-il le contraste entre un intime « tantôt perçu comme un petit monde clos, isolé, secret, propre à chacun, tantôt comme un infini extérieur en communication universelle ", c'est-à-dire une " conception romantique » de la notion ${ }^{4}$. L'ère industrielle, et l'élévation du niveau de vie, ont joué un rôle essentiel dans le déplacement des frontières de l'intime: avec l'apparition de ce 
qu'Antoine Prost appelle le "desserrement de l'espace domestique ${ }^{5}$ » (habitat privatif, chambre individuelle, salle de bains), l'intimité, en particulier corporelle, a changé de centre de gravité, se resserrant autour du corps. Mais l'individu, en parallèle, a vu les barrières de son moi devenir perméables à toutes sortes de sollicitations et d'injonctions, déversées dans son espace personnel par des médias que la technologie a su rendre invasifs. Nul ne s'aviserait, comme le faisaient certains rois, de passer aujourd'hui sa nuit de noces en public; mais de quel œil un homme du XvII ${ }^{\mathrm{e}}$ siècle aurait-il considéré la débauche de confidences sur les liaisons, la sexualité, la vie domestique qui font maintenant partie de la panoplie identitaire des personnalités publiques?

Si le phénomène varie au gré des époques, une constante demeure, fort bien résumée par Françoise Simonet-Tenant : « l'intime reste rebelle à la définition 6 . » Peut-être parce qu'il ploie, justement, sous trop de définitions. Son évolution sémantique est aussi complexe que sa réalité protéiforme ; l'évolution, voire la révolution lexicographique qui a frappé le terme est à l'aune de ses mutations sociales. Pour tenter de voir un peu plus clair dans la jungle de l'intime, et de distinguer les étapes charnières de ses changements d'acception, nous avons choisi d'observer le mot dans une double perspective diachronique. La première, lexicographique, s'appuie sur des définitions de dictionnaires, rédigées entre 1606 et 2008, et sur l'examen de leurs variations (souvent importantes); elle s'attache à mettre en évidence le déplacement du centre de gravité sémantique de l'adjectif. La seconde approche se fonde sur l'exploration de deux corpus: le catalogue général de la Bibliothèque nationale de France («BN-Opale Plus»), interrogé sur l'opérateur "titre", et la base de données Frantext, qui regroupe quatre mille textes français. À charge pour eux de confirmer ou d'infirmer la pertinence et la réactivité du discours lexicographique relativement à la notion d'intime.

\section{Parcours lexicographique}

4 La riche collection de documents lexicographiques de l'ATILF-CNRS' (Nancy) nous a permis de remonter aux sources de la présence du terme intime dans le lexique français. Cet adjectif désigne au départ un élément extérieur à la personne, puisqu'il est utilisé pour décrire une relation de proximité amicale particulièrement marquée. Avant le XVII siècle, intime est quasiment inexistant dans les dictionnaires de langue : le Huguet ne lui octroie d'ailleurs pas d'entrée, ne relevant qu'intimement, avec deux exemples traitant de l'amour de Dieu ou de Jésus. Le premier dictionnaire à lui accorder une définition est le Nicot (1606) : celle-ci calque l'étymologie (« Est ce qui est au profond \& en l'intérieur ») et l'illustre par l'exemple «l'amitié intime que j'ay à vous». Le Richelet (1679), donne du terme la définition suivante: "Mot qui vient du latin et qui signifie fort profond. Il se dit en françois des amis \& amies, \& veut dire qui est un particulier et vrai ami, qui est ami du fonds du cœur." Mais le Dictionnaire de l'Académie française, dans sa première édition (1694), spécialise d'emblée l'emploi du terme, en déclarant immédiatement après la vedette et le descripteur grammatical («adj. de tout genre ») : «Il n'a guère d'usage qu'en cette phrase. Ami intime, qui signifie, Un ami cordial, un homme avec lequel on a une liaison d'amitié très étroite. » 


\section{IN TIME. adj. đe tout genre. Il n'a guere d'u- fage qu'en cette phrafe. Ani intime, qui fi- gnifie, Un ami cordial, un homme avec lequel on a une liaifon d'amitié tres eftroite. En ce fens il eft quelquefois fubftantif. $C^{\prime}$ eft fon intime. Il eft du ftyle familier. On dit auffi union intime. liaifon intime.}

Fonds documentaire ATILF-CNRS Nancy.

Cette définition sera maintenue dans les quatre éditions suivantes, et sa réactualisation complète devra attendre 1835. Enfin Furetière, en 1701, concentre lui aussi la totalité de la définition sur la question relationnelle : "Ami particulier, \& a qui on descouvre son cœur, \& ses affaires plus confidemment qu'a tout autre. » Dans ces trois cas, la conscience ou les affects de l'individu ne sont pas évoqués ; le sème étymologique de l'intériorité, au lieu d'être entendu dans sa verticalité introspective, s'applique à une dimension latérale, à savoir la qualité du lien avec une tierce personne. Cette orientation lexicographique restreinte reste la dominante au XVIII ${ }^{\mathrm{e}}$ siècle: Trévoux (1704), se contente de décalquer Furetière pour la définition générale, mais y ajoute une acception religieuse :

Intime, en termes de Théologie, signifie aussi, Particulier, étroit, secret. Le but de la Théologie Mystique est de former une union immédiate, \& intime avec l'ame devote de Dieu.

Commence, avec les éditions successives de ce dictionnaire, un nouveau déploiement sémantique : d'abord par l'introduction d'acceptions particularisantes, qui marquent la migration de l'adjectif : « conseiller intime de l'Empereur », « liaison intime » (Supplément de 1752). Ensuite, en 1771, avec l'apparition d'une acception qualifiée de "métaphysique »; le sensus intimus est le "sentiment intérieur, ou conscience». La définition poursuit : «C'est ainsi que nous connaissons notre âme, les pensées, la douleur, le plaisir, en un mot tout ce qui se passe au dedans de nous-même. » En des termes plus contemporains, on pourrait dire que la psychologie fait son entrée dans le traitement lexicographique de l'intime. Même évolution du côté du dictionnaire de l'Académie, qui dans son édition de 1798, introduit un nouveau syntagme, persuasion intime, défini comme une « persuasion intérieure et profonde ». À ce titre, le XVIII ${ }^{\mathrm{e}}$ siècle constitue une sorte de transition sémantique: tout en maintenant au premier plan la problématique relationnelle, les lexicographes introduisent discrètement l'idée d'une intériorité du sujet et de sa conscience. Cet élément nouveau amorce un virage sémantique majeur, qui va ramener le terme à son acception étymologique.

5 Si Raymond (1832), décalqué par Boiste (1834), met encore au premier plan l'idée d'une " affection très forte » entre deux êtres, on peut considérer qu'en 1848, le tournant est consommé: Bescherelle (édition 1845-1846) donne comme premiers synonymes de l'adjectif intime "intérieur, profond», plaçant cependant la problématique sur un plan plus philosophique, voire scientifique, qu'affectif ou éthique : «se dit surtout de ce qui constitue l'essence d'une chose.» Cette acception va devenir la tête de pont du nouveau territoire sémantique de l'intime : elle constitue le sens 1 du Larousse du XIXe siècle (1873), 
et envahit ensuite tous les dictionnaires de la fin du XIx $x^{e}$ au début du $\mathrm{Xx}^{\mathrm{e}}$ siècle. Elle trouve par ailleurs de nombreuses illustrations dans divers titres d'ouvrages scientifiques proposant à leur lecteur la découverte et la compréhension de mécanismes physiques ou biologiques: Laënnec publie en 1856 ses Recherches sur le développement et la structure intime du tissu osseux, René Feret consacre un volume d'Études à la question de la constitution intime des mortiers hydrauliques (1898) tandis que Ludovic-Adrien Girondeau nous révèle les arcanes de La circulation cérébrale intime dans ses rapports avec le sommeil (1868).

6 La définition de Bescherelle, pour en revenir à elle, est ensuite complétée par quatre sousrubriques. La quatrième fait entrer, une fois de plus par la petite porte, la dimension individuelle dans la définition: «dans le sens intérieur, ce qui existe au fond de l'âme. Persuasion, conviction intime. Le sentiment intime de la conscience. » Peu à peu, l'idée de l'intériorité psychologique se ménage une place dans les dictionnaires; observation corroborée par le dictionnaire de Laveaux (1842), qui explicite sens intime par « sentiment de ce qui se passe au-dedans de nous ». On peut donc considérer que dès la première moitié du XIX ${ }^{e}$ siècle, le lien est fermement recréé, dans les définitions, entre la notion d'intime et sa dimension introspective. Le Dictionnaire historique de la langue française d'Alain Rey (édition de 1992) souligne l'adjonction de sens opérée sous l'influence du romantisme, qui associe l'intime à «ce qui est strictement personnel et généralement caché aux autres ", et notamment au secret. Et Littré, en 1873, synthétise les différentes acceptions, et hiérarchise la définition de la façon suivante : 1) plan physique (le plus en dedans et le plus essentiel), 2) plan moral (qui existe au fond de l'âme), 3 et 4) plans affectif et relationnel (très-étroit et très-cher), et 5) plan éthique (ce qu'il y a de plus profond dans une chose); une forte coloration religieuse est donnée à cette dernière section par les exemples de Pascal et Bossuet.

7 Les définitions $\mathrm{du} \mathrm{xx}^{\mathrm{e}}$ siècle, si elles reprennent classiquement l'étymologie, traitent en priorité de la dimension psychologique et ajoutent à leur tour de nouvelles acceptions, explicitant parfois des connotations dont la littérature portait trace depuis bien longtemps. La principale inflexion est un déplacement dans la hiérarchisation des "générateurs", si l'on peut dire, d'intime: non plus seulement les choses, mais les êtres. Et même : d'abord les êtres. Pour le Robert de 1959, l'intime est « ce qui est profondément intérieur, contenu au plus profond d'un être, lié à son essence; généralement secret, invisible, impénétrable ». Le Grand Larousse de la langue française (édition de 1975), place lui aussi la notion prioritairement du côté de l'humain («qui est au plus profond d'un être, d'une chose »). Le sens 1 est assorti d'une paraphrase d'exemple (« Sens intime, sentiment de notre vie psychique, conscience ») qui montre le déplacement définitif du concept vers des notions liées à la perception, au ressenti, aux affects, et de fait polarisées sur le sujet percevant. D'une certaine manière, est intime ce que je ressens comme tel. La cristallisation de ce nouveau sens est matérialisée par son passage dans les descripteurs du Grand Robert de la langue française (édition de 1985) : le secteur, littér., qui accompagne le premier sens, y est assorti d'une parenthèse : « (Domaine psychique, humain). »

8 Quant au Trésor de la langue française, sur lequel nous aurons à revenir, il opte, comme il en a l'usage dans le cas de notions complexes, pour une structure de définition fortement hiérarchisée : la tête de section ( $\mathrm{I} »)$ et sa première acception ( $\mathrm{A} »)$ mettent en valeur les caractères sémantiques considérés comme les plus saillants du lemme. En parfaite cohérence avec le Robert, le descripteur du champ notionnel couvert par I (« [en parlant d'une pers., de sa vie intérieure ou de ses rapports avec celle-ci]») insiste fortement sur le fait 
que l'intimité est avant tout recentrée sur l'individu. L'acception «A» commence par associer une terminologie moderne, dont il faudrait chercher les racines dans l'épistémologie médicale, et une dimension de verticalité, elle, étymologique: «qui se situe ou se rattache à un niveau très profond de la vie psychique. » Le TLF n'en reste pas là : comme ses homologues, il ajoute, dans sa définition, des sèmes que le $\mathrm{xx}^{\mathrm{e}}$ siècle, psychanalyse aidant, a fortement actualisés, avec des oppositions implicites entre conscient et inconscient: "qui reste généralement caché sous les apparences, impénétrable à l'observation externe, parfois aussi à l'analyse du sujet même. » La deuxième partie $(« \mathrm{II} »)$ de la définition, qui représente les deux tiers de celle-ci, se concentre sur les personnes, leur mode d'existence, leurs rapports avec autrui. En particulier, et parce que le dictionnaire offre une approche raisonnée qui se veut exhaustive des champs notionnels concernés, on y voit explicitement se dessiner le lien entre l'adjectif intime et la vie conjugale, ou la sexualité, entendue à divers degrés organiques : vie privée restreinte au couple, anatomie généralement cachée de quelqu'un, vie sentimentale ou sexuelle secrète de quelqu'un.

$9 \mathrm{Au} \mathrm{xx}^{\mathrm{e}}$ siècle, l'intime devient donc, en quelque sorte, l'espace d'expression d'une extériorisation limitée et (s)élective, de l'intériorité. Cette opération peut s'accomplir dans la privauté du journal, du couple, de la maison; la qualité d'intime en vient ainsi à désigner un périmètre autour de soi et ce que l'on considère comme relevant de soi, $\mathrm{y}$ compris au sens physique. Logiquement, la définition s'élargit et s'enrichit d'autant, à commencer par un accent mis sur le cercle relationnel. Le dictionnaire de Guérin esquisse, en 1892, l'idée de "famille intime », que reprend le Nouveau Larousse illustré de 1902. Pour ce dernier, est intime, "ce qui fait partie de la famille». On remarque également l'apparition de la notion de privauté, dans le Robert de 1959: «qui est tout à fait PRIVÉ, et généralement tenu caché aux autres. » Pour le Davau-Cohen (1972), la vie intime est "personnelle et familiale, dans le PRIVÉ ${ }^{8}$ ». L'introduction de ce terme est à proprement parler redondante, et même doublement si l'on se réfère à l'étymologie d' intime; mais le fait qu'il devienne nécessaire de qualifier l'adjectif en ce sens prouve que la frontière de l'intime est en train de se déplacer. L'espace de ce dernier s'élargit, et le rempart qui la protège - que le $\mathrm{xx}^{\mathrm{e}}$ siècle rendra bien poreux... - ne réside plus tant dans l'intériorité que dans la capacité à soustraire celle-ci à certains regards, tout en l'exposant volontiers à d'autres.

Ensuite, le périmètre d'application change de nature ; l'intime n'est plus seulement une question de for intérieur, mais s'applique aussi à certaines parties du corps. Le Quillet de 1965 parle de maladies intimes, données comme synonymes de «maladies des organes génitaux »; "on dit de même toilette intime ». Le Petit Larousse 2008, produit d'une dictionnairique tournée vers le grand public, c'est-à-dire plus synthétique, procède de manière identique, en fournissant un sens 4 qu'il se contente de définir par l'exemple : " Toilette intime: des organes génitaux.»Ces acceptions dépassent en réalité largement le périmètre des ablutions et vont de pair avec une association, implicite depuis des siècles dans les dictionnaires, entre l'intime et la sexualité. Le Nouveau Larousse illustré de 1902 précisait déjà, et pour une fois sans ambages : «Relations intimes, Rapports sexuels »; le Robert (1959): «Vie intime, celle que les autres ignorent, notamment la vie sentimentale, sexuelle. » La définition du TLF a perçu de façon claire cette nouvelle manière de penser le corps comme objet d'intimité, et en a formalisé de manière cohérente la définition, par une transposition (sens II.2.a.) du domaine moral au domaine anatomique: "Qui appartient à la vie physique la plus secrète, à l'anatomie généralement cachée de 
quelqu'un.» La suite de la section (sens II.2.) précise le lien avec la sexualité : «qui concerne la vie sentimentale ou sexuelle de quelqu'un", et "en partic.», "qui se manifeste, s'exerce par un contact charnel étroit, par une union sexuelle». Hors les dictionnaires, il est fréquent de trouver l'adjectif intime utilisé dans ce sens précis, pour euphémiser la sexualité, ou même la pornographie : c'est ainsi qu'il nous a été donné, dans le très sérieux catalogue général de la Bibliothèque nationale de France, d'identifier une collection, «Intime vidéo », produite par Marc Dorcel, et dont les trois titres - nous préférons en faire grâce au lecteur-laissent sans ambiguïté comprendre de quelle catégorie cinématographique et de quel intime il s'agit.

11 On peut donc résumer brièvement le parcours d'intime: d'un sens restreint, et uniquement appliqué à un phénomène extérieur au sujet - une relation de confiance, d'amour ou d'amitié - il revient progressivement à sa racine étymologique pour qualifier une intériorité. Celle des éléments et des corps biologiques, d'abord, dans leur structure et leur nature physico-chimique, puis celle des êtres. La dimension religieuse, éthique, ontologique prise en compte à partir $\mathrm{du} \mathrm{xVIII}^{\mathrm{e}}$ siècle pour définir l'intimité élargit considérablement son spectre; en même temps, l'une de ses acceptions se tourne plus précisément vers l'idée de secret et de privauté, en particulier dans le domaine sentimental. Le $\mathrm{xx}^{\mathrm{e}}$ siècle lui ajoute une coloration psychologique, voire psychanalytique, qui place la notion en lien étroit avec le ressenti du sujet. Et une nouvelle spécialisation, à l'intérieur de l'isotopie de l'intime, englobe désormais les relations de couple, la perception du corps et la sexualité. Cette évolution se fait toujours dans le sens d'une amplification; comme le note Jean Beauverd, «si l'évolution a multiplié les acceptions, elle n'en a détruit aucune ${ }^{9} »$.

\section{Enquête statistique}

Une fois récapitulées les principales évolutions du terme intime, voyons si son utilisation en langue corrobore les descriptions lexicographiques qui en ont été faites. Pour apprécier le comportement sémantique de ce lemme, nous avons recouru à son examen dans deux bases de données informatisées. La première est le catalogue général de la Bibliothèque nationale de France, mieux connu sous le nom de "BN-Opale Plus", qui recensait en février 2009 plus de sept millions de notices. Nous l'avons interrogé, à l'aide de diverses méthodes (recherche simple, recherche complexe, équation de recherche) pour en extraire, siècle par siècle, tous les titres d'ouvrages (imprimés et manuscrits seulement, hors titres de collection et ouvrages étrangers) contenant le mot intime. Le moteur de recherche de BN-Opale Plus ne fait pas la différence entre caractères accentués et non accentués: la présence de l'adjectif «intimé », présent dans des milliers de documents judiciaires, a donc créé un bruit important, qui a nécessité une première sélection. Ensuite s'est posé le problème des rééditions, certes significatives (en termes de succès commercial, de mode, d'imaginaire social), mais qui parasitaient les calculs statistiques de co-occurrences: nous les avons donc éliminées. Cette double sélection nous a permis de conserver 1410 des 7350 réponses obtenues. Cette liste de titres forme un premier corpus : même si elle ne concerne pas des textes intégraux et rédigés, elle permet tout de même d'observer des comportements morphosyntaxiques (substantivation, anté ou post-position de l'adjectif, co-occurrences avec tel ou tel substantif) qui rendent son observation pertinente sur un plan linguistique. 
13 Le deuxième outil utilisé est la base de données Frantext, qui regroupait 3911 textes français et francophones en février 2009. Cette fois l'interrogation a été faite sur le contenu, et non plus seulement sur les titres, des ouvrages. Frantext ne possède pas la même représentativité que le catalogue de la BNF : sa mission n'a jamais été de servir de bibliothèque numérique exhaustive ou étendue, à la façon de Gallica ou de Google Livres, mais plutôt de fournir des outils de recherche perfectionnés sur une sélection d'ouvrages considérés comme représentatifs de la littérature française : ceux-là même qui avaient été choisis pour alimenter le corpus d'exemples du Trésor de la Langue Française. La possibilité de rechercher les co-occurrences, les formes lemmatisées, les listes de fréquence, les voisinages, et surtout l'affichage de contextes de 350 signes autour de l'occurrence permet d'obtenir des résultats plus riches, qu'il est possible de contraster les uns par rapport aux autres, en diachronie comme en synchronie.

14 Le premier constat, d'ordre quantitatif, permet de voir qu'au fur et à mesure que le mot intime élargit ses emplois, sa représentativité progresse dans la langue. D’abord quasi inexistant dans le catalogue des titres des publications (une occurrence seulement au XVI siècle, quatre $a u X V I I^{e}$, cinq $\left.a u X V I I I^{e}\right)$ il reste cantonné à la qualification d'une relation avec une personne («D'après la lettre signée de foi D'UN AMI INTIME », « Copie d'une Lettre confidentielle envoyée de Sibérie À UN AMI INTIME»), ou un groupe ("Théâtre de l'Hermitage de Catherine II, composé par cette princesse, par plusieurs personnes de sa SOCIÉTÉ INTIME et par quelques ministres étrangers ${ }^{10} \ldots$...). Seul l'un des titres fait apparaître un syntagme lié à l'intériorité, avec la notion de conviction (Matthieu Villenave, Des jurés et de la conviction intime, 1795).

Présence du mot « intime » dans BN-Opale Plus et Frantext

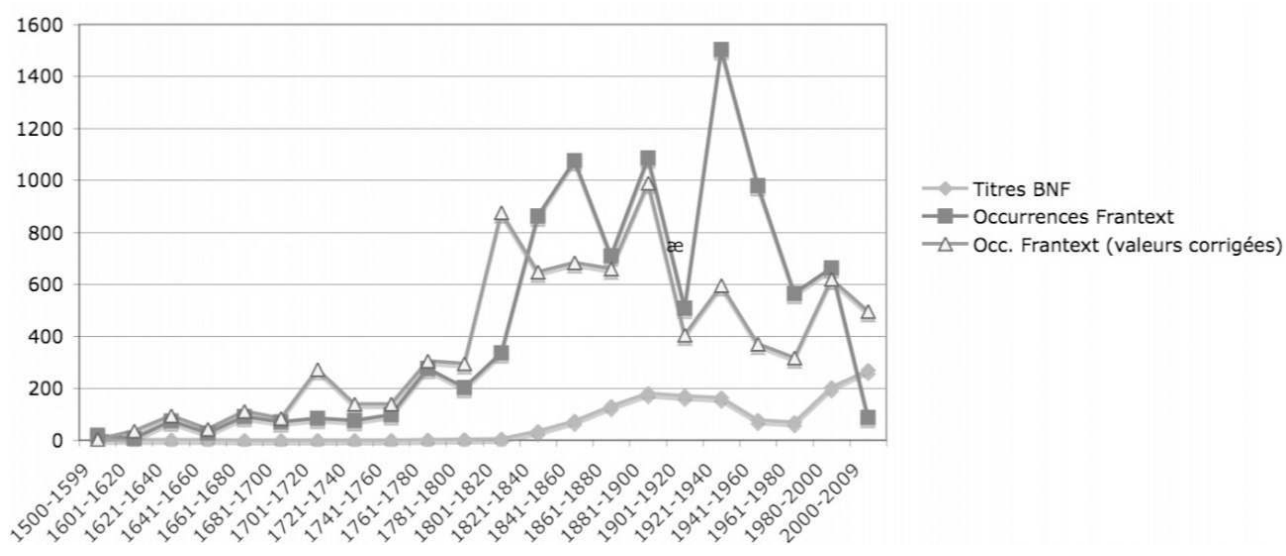

Frantext permet de mieux apprécier cette évolution ${ }^{11}$. On y trouve une confirmation globale de la tendance détectée dans les titres, mais avec un rythme de croissance bien plus marqué et bien plus rapide: si l'on ne repère que 17 occurrences d'intime dans les textes du XVI ${ }^{\mathrm{e}}$ siècle, le XVII ${ }^{\mathrm{e}}$ en offre près de seize fois plus, avec 269 occurrences, et le $\mathrm{XVIII}^{\mathrm{e}}$, quarante-trois fois plus, avec 738 occurrences. On peut expliquer cette différence d'augmentation entre fréquences dans les titres et fréquences dans les textes par deux hypothèses : de toute évidence, avant le XIX ${ }^{\mathrm{e}}$ siècle, l'intime n'est pas encore un objet de préoccupation ou d'intérêt social suffisant pour que le titre, "nom propre d'un livre ", comme le dit fort bien Jacques Roubaud, juge pertinent d'y faire référence. D'autre part, sur le plan lexical, le sens de l'adjectif est encore spécialisé, ce qui restreint la palette des substantifs auxquels on peut l'appliquer, et partant, des titres possibles. Ce que nous 
confirme l'observation du mot en contexte, qui montre comment, nonobstant l'augmentation des scores, le périmètre d'emploi reste délimité. Au XVII ${ }^{\mathrm{e}}$ siècle, la cooccurrence vedette est celle d'ami, sous deux graphies (ami et amy), qui monopolise plus de $75 \%$ des voisinages!

On remarque également que la montée en charge des occurrences tend à s'accélérer dans la seconde moitié du XviII ${ }^{\mathrm{e}}$ siècle, dans les titres en particulier: plus des deux tiers de ceux qui comprennent le mot intime $(73 \%)$ sont publiés postérieurement à 1760 . Cette situation recoupe les constats opérés par ailleurs par les historiens, dont certains situent la naissance de l'intime entre la fin du XVIII et le début du XIX ${ }^{e}$ siècle, sous l'influence conjointe de la démographie, de la mobilité sociale, de la concentration urbaine et des bouleversements qu'introduit la Révolution française. Alain Girard postule qu'après avoir «renversé toutes les valeurs établies, les ordres, les classes sociales, Dieu, les règles de l'art », il ne reste à l'individu " qu'un seul absolu, le plus fragile de tous, le moi, ou qu'un seul refuge, l'intimité, triomphante ou modeste ${ }^{12} »$. Annick Pardailhé-Galabrun, qui a dépouillé les inventaires après décès du Minutier central des notaires, note qu'au XVIII siècle, l'intimité telle que nous la comprenons n'apparaît que timidement dans les objets domestiques (par exemple les accessoires de toilette ou de bain), et que sa conquête « ne sera réalisée qu'au XIX ${ }^{e}$ siècle $^{13}$ ».

De cette conquête, les textes témoignent, en effet, avec une véritable explosion des emplois du terme. Ce ne sont pas moins de 426 ouvrages, publiés entre 1801 et 1900, qui comprennent le mot « intime » dans leur titre, soit une proportion qui centuple le chiffre du siècle précédent. Frantext enregistre, quant à elle, une croissance de... $555 \%$ des occurrences, avec plus de 4000 emplois. L'acmé est atteint entre 1881 et 1900, avec 1087 occurrences Frantext (VA), ce qui représente aussi, en valeur relative, le pic le plus haut de l'ensemble de la période considérée; même tendance du côté des titres relevés dans BN-Opale, qui enregistrent également l'un des scores les plus élevés (181 références) pour ces deux décennies. $\mathrm{Au} \mathrm{Xx}{ }^{\mathrm{e}}$ siècle, une présence forte du terme se maintient dans les deux corpus considérés, avec toutefois un nouveau profil de la courbe : intime devient moins fréquent dans les titres d'ouvrages, avec un decrescendo lent, puis une chute pour la période 1941-1980, avant une remontée en flèche à partir de 1980. On peut attribuer cette désaffection légère à diverses causes : fin d'une vogue lexicale, qui a vu le terme intime employé pour qualifier toutes sortes de situations et de personnages différents; événements historiques, puis politiques, qui ont remis au premier plan des problématiques collectives. 


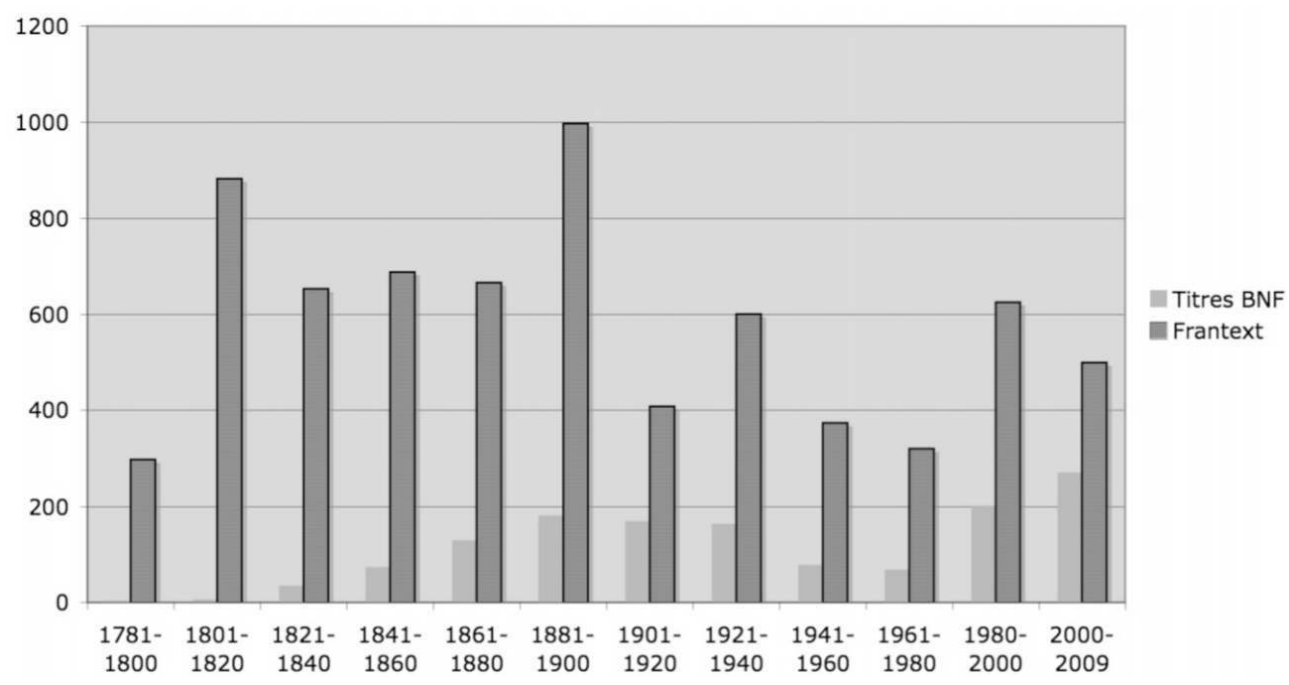

Dans le corpus de Frantext, la situation est moins tranchée, puisque le score remonte durant l'entre-deux-guerres (600 occurrences VA entre 1921-1940) mais qu'ensuite, la courbe décroît également, jusqu'en 1980. À ce moment-là apparaît un nouveau pic, et dans les titres et dans les textes (remontée à 202 titres et 663 occurrences VA), qui semble être une tendance lourde du xxI ${ }^{e}$ siècle naissant. Certes, le corpus contemporain présent dans Frantext est susceptible d'introduire un biais, du fait d'une opération ciblée d'enrichissement de textes autobiographiques menée depuis 2006. Cela dit, les titres de la BNF confirment cette tendance, puisque pour la période 2001-2008, les scores d' « intime » égalent ou dépassent ceux des vingt années qui précèdent. Au reste, on peut constater de manière plus empirique ce succès en observant les tables de librairie ou en effectuant quelques requêtes chez Amazon.com - qui commercialise même un logiciel permettant de tenir son journal intime ${ }^{14}$ !

Les deux bases nous permettent donc de constater, pour les années 1980, une situation comparable à celle de la fin du XIX $x^{e}$ siècle, à savoir un effet de mode, récupéré par la machine éditoriale, et renforcé par le fait qu'intime s'est entre-temps chargé de sens multiples, notamment en périphérie des domaines de la psychologie et de la sexualité. Le lecteur appréciera ainsi de pouvoir feuilleter Star Academy, l'album intime (2003) - même si l'on se demande ce qui peut rester d'intime à quiconque ayant subi l'épreuve d'une téléréalité; de bénéficier de recommandations éducatives (Véronique Mahé, Les 100 premiers jours avec bébé: journal intime d'une jeune maman et les précieux conseils d'une pédiatre, 2005) ou d'en apprendre davantage sur (toutes) les prouesses des champions avec Le Sexe et le Sport : enquête sur la vie intime des dieux du stade (Tristan Alric, 2002).

\section{Qu'est-ce qui est intime dans l'intime?}

Les objets auxquels s'applique l'adjectif intime peuvent nous renseigner plus avant sur la teneur de la sphère dans laquelle cette notion est, ou devient, opératoire. Nous avons donc relevé dans le catalogue BN-Opale Plus, et fait relever par Frantext, qui possède un outil dédié (la recherche de voisinage), tous les mots - essentiellement des substantifs et des noms propres -, auxquels s'appliquait l'adjectif intime, ainsi que ses emplois substantivés. Nous les avons ensuite triés et classés par ordre décroissant de fréquence, 
obtenant ainsi une sorte de cartographie du vocabulaire avec lequel intime développe une affinité. Pour améliorer la lisibilité du résultat, nous avons exprimé les scores de chaque lemme, dans chaque siècle, en \%. Par exemple, si le mot conversation est associé douze fois à l'adjectif intime dans les titres d'ouvrage du $\mathrm{xx}^{\mathrm{e}}$ siècle, soit dans $4,4 \%$ des cas, nous sommes en mesure d'évaluer qu'il est bien plus représenté que confessions ( 2 occurrences, $0,7 \%$ ), mais bien moins que vie (92 occurrences, $34 \%$ ). Pour clarifier encore l'appréciation, nous avons identifié dans chaque liste de co-occurrents dix paliers de fréquences, qui découpent l'ensemble en secteurs de plus ou moins forte pertinence : la zone 1 comprendra les $10 \%$ des lemmes les plus représentés, la zone 2 les $10 \%$ suivants, etc. jusqu'à la zone 10, qui ne comprend en général que des hapax. Pour reprendre notre exemple, vie se trouve en zone 1-2 (à cheval sur les deux paliers), sa pertinence de fréquence est haute; conversation est en zone 4 et confessions en zone 8 , soit une pertinence faible (ce qui ne signifie pas sans intérêt). Ce mode d'affichage des résultats nous sera utile au moment de comparer, en diachronie, à quels autres mots (les « voisins ») est associé le terme intime.

\section{Termes co-occurrents}

$21 \mathrm{Au} \mathrm{XVI}^{\mathrm{e}}$ et au XVII siècle, comme le parcours lexicographique nous l'a montré, le terme intime reste d'un emploi restreint, et n'est accolé qu'à une série limitée de substantifs. Dans Frantext, son plus proche voisin (sans doute l'était-il parfois au sens propre du terme !) est le substantif ami: au xVI ${ }^{e}$, il représente $756,2 \%$, soit les trois quarts des termes co-occurrents, et mobilise les zones 1 à 8 , ce qui est tout à fait exceptionnel. Les autres fréquences, comparées à cette valeur, sont quasi-marginales (la suivante, certitude, ne représente plus que $16,6 \%$ ) et se distribuent sur 45 co-occurrents seulement. Même tendance au XVII ${ }^{\text {e }}$ siècle, où ami tient toujours la corde, avec une large avance sur ses concurrents : $322 \%$, soit environ le tiers des occurrences, et le recouvrement des zones 1 à 3. La distribution du reste de la liste, qui comporte davantage de termes co-occurrents, est moins déséquilibrée, mais continue à marquer une certaine étroitesse du périmètre sémantique : les premiers voisins qui suivent directement ami ont des valeurs là aussi bien plus basses (de 74 à $11 \%$ ) : on relève liaison, sentiment, commerce, union, conviction, société, nature, confiance, correspondance, structure, sens, connaissance, conscience, persuasion, familiarité, confidence, amitié. Soit, dans la majeure partie des cas, une utilisation d'intime comme descripteur qualitatif d'une relation, d'une attitude avec autrui-toujours en situation adjectivale. Le catalogue de la BNF, bien que ne fournissant qu'un nombre restreint de titres pour cette période, confirme cette hégémonie : ami intervient dans le sous-titre de l'unique référence $\mathrm{du} \mathrm{XVI}^{\mathrm{e}}$ siècle («ceste presante confession laquelle luy auait esté enuoyée de la ville de Bono en Nauarrois, par ledit S. d'Espernon, son-tres intime amy ») et dans deux des quatre titres du xviI (les deux autres évoquant un conseiller intime).

$22 \mathrm{Au} \mathrm{XIX}^{\mathrm{e}}$ siècle, la situation se présente quelque peu différemment, et le contenu des titres et des textes commence à diverger. Nous indiquons en caractères gras les substantifs communs aux zones de pertinence 1 à 4 .

\begin{tabular}{|l|l|l|l|l|l|l|l|}
\hline Frantext XIX (1801-1990) & Occ. & \%o & Zones & $\begin{array}{c}\text { Titres } \\
\text { BN-Opale Plus }\end{array}$ & Occ. & $\%$ & Zones \\
\hline
\end{tabular}




\begin{tabular}{|c|c|c|c|c|c|c|c|}
\hline ami & 386 & 129,0 & 1 et 2 & vie & 107 & 257,8 & 1 à 3 \\
\hline vie & 127 & 42,4 & 2 & histoire & 27 & 65,1 & 4 \\
\hline sentiment & 82 & 27,4 & 2 & correspondance & 23 & 55,4 & 4 \\
\hline pensée & 64 & 21,4 & 3 & journal & 17 & 41,0 & 5 \\
\hline sens & 57 & 19,0 & 3 & drame & 13 & 31,3 & 5 \\
\hline secrétaire & 56 & 18,7 & 3 & secrétaire & 10 & 24,1 & 5 \\
\hline rapport & 54 & 18,0 & 3 & structure & 9 & 21,7 & 5 \\
\hline conviction & 52 & 17,4 & 3 & ami & 8 & 19,3 & 6 \\
\hline union & 49 & 16,4 & 4 & conseiller & 8 & 19,3 & 6 \\
\hline relation & 44 & 14,7 & 4 & roman & 8 & 19,3 & 6 \\
\hline connaissance & 33 & 11,0 & 4 & poésie & 6 & 14,5 & 6 \\
\hline confidence & 32 & 10,7 & 4 & revue & 6 & 14,5 & 6 \\
\hline causerie & 32 & 10,7 & 4 & souvenir & 6 & 14,5 & 6 \\
\hline journal & 29 & 9,7 & 4 & constitution & 5 & 12,0 & 7 \\
\hline nature & 28 & 9,4 & 4 & édition & 5 & 12,0 & 7 \\
\hline liaison & 28 & 9,4 & 4 & réunion & 5 & 12,0 & 7 \\
\hline conversation & 28 & 9,4 & 4 & théâtre & 5 & 12,0 & 7 \\
\hline
\end{tabular}

Comme on le voit, ami est toujours en tête dans les textes, mais il ne représente plus que $129 \%$ des emplois, et ne mobilise plus « que » les zones 1 et 2 de la liste. La distribution des autres occurrences se fait cette fois sur 720 autres substantifs et noms propres, ce qui confirme son expansion polysémique. Les orientations les plus marquées-nous indiquons entre parenthèses leur nombre d'occurrences, suivi du numéro de leur zone de pertinence -, sont :

- la sphère de la conscience et des intuitions : sentiment (82-2), pensée (64-3), sens (57-3), conviction (52-3), connaissance (33-4) ;

- les relations humaines (et intellectuelles) et leur structuration, avec l'expression de liens d'une personne à l'autre, ou bien noués au sein de groupes ou d'éléments : rapport (54-3), union (49-4), société (27-5), lien (-5), réunion (12-6), alliance (13-6), correspondance (11-6), liaison (28-4). Ce dernier terme, qui à l'heure contemporaine connote fortement la relation amoureuse, désigne au $\mathrm{XIX}^{\mathrm{e}}$ siècle des liens autrement plus abstraits: liaison intime «de deux idées analogues » (Destutt de Tracy), de "toutes nos facultés » (Benjamin Constant), «entre l'esprit de famille et la conservation de la terre » (Tocqueville), de la «mer» et des « rouages célestes » (Balzac); 
- le sens étymologique, celui de l'intériorité et la composition interne : nature (28-4), structure (17-5), constitution (12-6);

- le réseau lexical de la parole, qui suggère qu'au XIx ${ }^{\mathrm{e}}$ siècle, l'intime n'est pas cantonné au secret $\mathrm{du}$ for intérieur, mais peut faire l'objet d'un partage verbal: causerie (32-4), conversation (28-4), entretien (15-5);

- les affects et les relations amoureuses : joie (18-5), satisfaction (16-5), amitié (14-5), bonheur (13-6), confiance (13-6), émotion (12-6), sympathie (11-6) ;

- le secret : confidence (32-4), secret (17-5), confident (16-5). en deux secteurs : celui, prédominant, de l'intellect, du rationnel, qui utilise l'intime comme outil de connaissance, pour aller au cœur des choses et de la conscience, sans forcément le colorer d'affect. L'autre versant, lui, s'attache davantage à la dimension émotionnelle, qu'il ouvre sur un partage restreint et électif. Nous y reviendrons ultérieurement, mais le journal intime, qui prend son essor au $\mathrm{XIX}^{\mathrm{e}}$ siècle, est le parfait symbole de cet équilibre subtil entre intériorité, privauté, et extériorisation de soi. Du côté du catalogue de la BNF, les lemmes co-occurrents des titres et des sous-titres, eux, insistent davantage sur la dimension biographique (vie, histoire, correspondance) et générique. On peut ainsi remarquer que l'adjectif intime fonctionne comme une étiquette de caractérisation polyvalente qui s'applique à tous types d'écrits : drame, histoire, album, roman, comédie, revue, scène, causerie, épisode, carnet, poésie, relation, allocution, et même un " griffonnage intime ». Cet usage est loin de faire l'unanimité : le dictionnaire de JournetPetit, en 1967, le condamnera par une citation de F. Wey (Remarques sur la langue française au dix-neuvième siècle, 1945), déclarant à propos des syntagmes romans intimes ou genre intime : « cette façon de parler est "tout à fait dans le style des Précieuses ridicules et on la repoussera tant que l'affectation et la recherche seront considérées comme des défauts".»

Le $\mathrm{xx}^{\mathrm{e}}$ siècle marque un nouveau tournant, qui pour être moins spectaculaire que le précédent, n'en reflète pas moins un nouveau déplacement des polarités. La répartition gagne en homogénéité : les co-occurrents sont plus nombreux et plus diversifiés encore, dans les deux bases : 1007 pour Frantext, 263 pour BN-Opale Plus.

\begin{tabular}{|l|r|l|l|l|r|c|c|}
\hline $\begin{array}{c}\text { Frantext } \\
1901-2000\end{array}$ & Occ. & \%o & Zone & $\begin{array}{c}\text { Titres } \\
\text { BN-Opale Plus }\end{array}$ & Occ. & $\%$ Zone \\
\hline journal & 192 & 70,5 & 1 à 2 & journal & 221 & 331,3 & 1 à 4 \\
\hline vie & 92 & 33,8 & 2 & vie & 71 & 106,4 & 4 à 5 \\
\hline ami & 58 & 21,3 & 2 & correspondance & 15 & 22,5 & 5 \\
\hline nature & 44 & 16,2 & 2 & carnet & 10 & 15,0 & 5 \\
\hline sentiment & 40 & 14,7 & 2 & conviction & 8 & 12,0 & 5 \\
\hline relation & 39 & 14,3 & 2 & ennemi & 7 & 10,5 & 5 \\
\hline contact & 35 & 12,9 & 3 & histoire & 7 & 10,5 & 6 \\
\hline
\end{tabular}




\begin{tabular}{|l|r|r|l|l|r|r|l|}
\hline pensée & 35 & 12,9 & 3 & ami & 4 & 6,0 & 6 \\
\hline union & 33 & 12,1 & 3 & Carlyle & 4 & 6,0 & 6 \\
\hline sens & 32 & 11,8 & 3 & chronique & 4 & 6,0 & 6 \\
\hline rapport & 31 & 11,4 & 3 & constitution & 4 & 6,0 & 6 \\
\hline expérience & 27 & 9,9 & 3 & étranger & 4 & 6,0 & 6 \\
\hline drame & 26 & 9,5 & 3 & nature & 4 & 6,0 & 6 \\
\hline structure & 26 & 9,5 & 3 & Paris & 4 & 6,0 & 6 \\
\hline écrits & 24 & 8,8 & 3 & Anna Pavlova & 3 & 4,5 & 6 \\
\hline liaison & 21 & 7,7 & 3 & Calder & 3 & 4,5 & 6 \\
\hline connaissance & 20 & 7,3 & 3 & cercle & 3 & 4,5 & 6 \\
\hline conviction & 20 & 7,3 & 3 & cour & 3 & 4,5 & 6 \\
\hline espace & 20 & 7,3 & 3 & femme & 3 & 4,5 & 6 \\
\hline & & & & guide & 3 & 4,5 & 6 \\
\hline & & & & Huysmans & 3,5 & 6 \\
\hline
\end{tabular}

Les textes nous renvoient un profil sémantique qui ne diffère pas fondamentalement de celui observé au siècle qui précède, mais quelques évolutions sont perceptibles :

- une plus grande importance accordée aux outils d'écriture, à mettre en rapport avec le succès de la pratique diaristique : journal (192-1), écrits (24-3), carnet (12-4), cahier (7-5), lettre (5-6), papiers (5-6);

- une présence, nouvelle, du corps physique : toilette (10-4), odeur (9-4), chaleur (8-5), voix (65), hygiène (3-7). Cette tendance se trouvait déjà attestée par quelques occurrences entre 1801 et 1900 et renseigne sur l'évolution qui s'est opérée entre le XIX et le $\mathrm{XX}^{\mathrm{e}}$ siècle, qui a agrégé l'organique au champ de signification de l'intime. Cette époque charnière voit la découverte de l'asepsie et l'émergence de principes hygiénistes (favorisés par l'installation de salles de bains dans les habitats bourgeois), qui conduisent à promouvoir de nouvelles pratiques, dont les titres du catalogue de la BNF portent la trace. On relève ainsi, sous la plume du $\mathrm{D}^{\mathrm{r}}$ Marie de Thilo, un Livre de la mère de famille, sous-titré L'hygiène intime, conseils aux femmes pour tous les âges de la vie (1892) ; puis, publié sous le nom d'une certaine « Armide », L'Hygiène intime de la jeune fille, la femme, l'enfant (1921). Le $\mathrm{D}^{\mathrm{r}}$ Dupuytrem, quant à lui, offre un Petit traité théorique et pratique de la toilette intime et des maladies de femmes (1904). Certes, il existe un ouvrage plus généraliste (Conseils à tout le monde. Conférences sur l'hygiène intime), proposé par le $\mathrm{D}^{\mathrm{r}}$ Constant Poignet en 1871, mais dans l'ensemble, ces traités rendent 
palpable la suspicion qui porte sur le corps féminin, perçu comme un réservoir potentiel d'impuretés et de germes ;

- l'évocation explicite de la sexualité et de la génitalité, elle, existe, mais elle reste assez minoritaire sur le plan lexicométrique. Rien dans les zones de pertinence élevée n'y renvoie : les deux occurrences de délices et de caresses n'ont que métaphoriquement à voir avec des contacts charnels ; les trois de pénétration concernent uniquement des questions de géographie humaine ou d'intellection. En revanche, on trouve de nombreux exemples isolés parmi les hapax. Marie Chaix dans un effet d'hypallage, parle de «l'intime de la culotte [qui] devait à tout prix être dissimulée aux regards indiscrets ${ }^{15}$ » durant l'enfance, manière euphémistique d'éviter de nommer le sexe. Violette Leduc, dans la description d'une scène étrange chez le coiffeur, utilise une image volontairement ambiguë, qui évoque un tout autre genre de caresses que les soins capillaires : «Bouches, lèvres ourlées pareilles à des guêpes taquinèrent, emmêlèrent les fils de ma toison intime ${ }^{16}$.» Gabriel Matzneff, moins lyrique, mentionne lui un tube de « lubrifiant intime » acheté par un personnage "dans un sex-shop d'Amsterdam ${ }^{17} »$.

\section{Nominalisation}

Sur le plan grammatical, la substantivation de l'intime comme concept est de plus en plus fréquente à partir du XIX ${ }^{e}$ siècle, où l'on en relève vingt-quatre exemples dans Frantext. Cet emploi semble encore assez nouveau, puisqu'il ne concerne qu'un nombre restreint d'auteurs, dont certains, toutefois l'affectionnent tout particulièrement. Telle Eugénie de Guérin qui, dans son Journal (1838-1840) et ses Lettres (1831-1847) en fait un large usage : on en trouve onze emplois sous sa plume, avec une certaine variété dans le choix des prépositions : "c'est DE l'intime», «morte pour mon cahier, pour toi, POUR l'intime ", "vous me voyez [...] JUSQU'À l'intime», et enfin, l'intéressante locution "faire DE l'intime ", comme synonyme de pouvoir s'entretenir discrètement (en l'occurrence, avec une amie), ou encore « s'entendre À l'intime ». Bloy, Hugo, Bourget, les Goncourt et Péladan l'utilisent également, avec une expansion nominale : «l'intime de mon être ${ }^{18}$ », «l'intime de ma pensée ${ }^{19}$ ", "l'intime de mon âme ${ }^{20}$ ». Au xxe siècle, les occurrences doublent : 48 exemples dans Frantext. Là aussi, le terme peut être employé dans l'absolu: «on ne se demande rien sur l'intime ${ }^{21}$ », "f[aire] primer [...] l'intime et le substantiel ${ }^{22}$ ", «l'adaptation de l'intime à l'extérieure réalitée ${ }^{23}$ ". Valéry, qui affectionne le terme, aime à l'employer sous cette forme ( une infernale combinaison du sacerdoce et du négoce, de l'intime et de la publicitée $\left.{ }^{4} »\right)$, mais on le retrouve aussi bien sous la plume de Paul Ricœur, Gaston Bachelard, Louis Aragon ou André Gide («Tout l'intime demeure un mystère $\left.^{25} »\right)$. De plus en plus d'auteurs l'utilisent eux aussi sous sa forme substantivée assortie d'un complément de nom, preuve de la naturalisation définitive du terme en tant que concept: Proust parle de «l'intime de sa [la femme] vie de tous les jours ${ }^{26} »$, SaintJohn Perse évoque «tout l'intime de l'eau [qui] se resonge en silence aux contrées de la toile ${ }^{27}$ ", Aragon "l'intime des draps ${ }^{28}$ ». Dans ce cas, le changement de classe ramène directement à l'étymologie, puisque dans ces différents exemples, il s'agit d'atteindre une sorte de moelle, de quintessence, d'absolue intériorité. Gabriel Matzneff, dans un beau pléonasme étymologique, parle au demeurant, en évoquant un personnage qui ne peut se mentir à lui-même de «l'intime de son intime ${ }^{29}$ ». Cette recatégorisation grammaticale, dans une certaine mesure, développe un effet néologique ${ }^{30}$ : de qualification d'un élément extérieur (personne, relation, objet), l'intime gagne une qualité d'abstraction et un contenu notionnel. Ce nouvel emploi gagne les titres des ouvrages : encore absent au XIX ${ }^{e}$ 
siècle $^{31}$ dans BN-Opale, l'intime substantivé se retrouve dans vingt-quatre titres entre 1901 et 2000. Plusieurs de ces ouvrages sont d'ailleurs des études théoriques sur la question : L'Intime : études sur l'objet (François Baudry, 1988), Secrets, secrets : de l'intime au collectif (Gilbert Maurey, 1999), Les Écritures de l'intime : la correspondance et le journal (2000), actes d'un colloque organisé à Brest en 1997 sous la direction de Pierre-Jean Dufief.

\section{Journal intime}

Le syntagme "journal intime » et son évolution méritent un examen particulier parce qu'ils correspondent à une réalité particulière. Le journal est une pratique, comme l'a montré Philippe Lejeune, qui a progressivement accédé au statut de genre, y gagnant au passage une légitimité littéraire et une visibilité éditoriale: une "vigne vierge proliférante » devenue " "variété" cultivée, produite en serre ${ }^{32}$ ». Ce faisant, il a en grande partie perdu son caractère "intime " au sens d'intérieur, secret, caché. Et l'émergence du néologisme «extime», popularisé par Michel Tournier en $2002^{33}$, reflète bien toute l'ambivalence qui régit désormais ce type de texte, rendu plus public encore par l'explosion des blogs. C'est pourquoi si Pierre Pachet maintient le terme d'intime pour les journaux, Philippe Lejeune, Jacques Lecarme et Françoise Simonet-Tenant préfèrent lui substituer le qualificatif de " personnel », mieux à même de rendre compte de la diversité des textes regroupés sous cette appellation ${ }^{34}$. Sur le plan lexicographique, journal intime n'existe pas dans les dictionnaires avant le $\mathrm{xx}^{\mathrm{e}}$ siècle, ce qui témoigne d'un important décalage par rapport à la pratique. En effet, l'on sait, notamment grâce aux travaux de Philippe Lejeune, que le début du journal dit « intime » en France est sans doute antérieur à la Révolution, et que ceux des jeunes filles prennent leur essor dès $1850^{35}$; les historiens du genre, en fixent de manière convergente les débuts vers $1780^{36}$ et « l'épanouissement ${ }^{37}$ » au XIX ${ }^{\mathrm{e}}$ siècle.

Or, si le Robert Historique de la langue française (édition de 1992), fait bien remonter l'expression à 1780 , et propose 1816 comme première datation de "journaux intimes ", dans les dictionnaires de langue, cet emploi est absent: de Raymond (1832), de Boiste (1834), de Littré (1867), du Dictionnaire de l'Académie française (1878), de Guérin (1892), du Larousse du XIX $X^{e}$ siècle (1873). En réalité, il faut attendre le Robert de 1959 pour en trouver trace : «Se dit d'écrits autobiographiques qui touchent à la vie privée d'un auteur et qu'il ne destine généralement pas à la publication." Dubois (1967) range pour sa part l'exemple du journal sous la rubrique « qui est caché des autres et appartient à tout ce qu'il y a de tout à fait privé », et en donne pour synonyme « journal secret ».

$\mathrm{Au}$ moment où elles paraissent, ces deux définitions vont déjà à contresens de l'évolution réelle de la pratique qui tend à être de moins en moins secrète, et de plus en plus tournée vers la publication ${ }^{38}$. En termes lexicométriques, l'évolution de l'expression journal intime est constante, mais irrégulière : on relève deux pics, les périodes 1921-1940 et 1980-2000, avec une évolution globale cohérente entre titres et textes. 


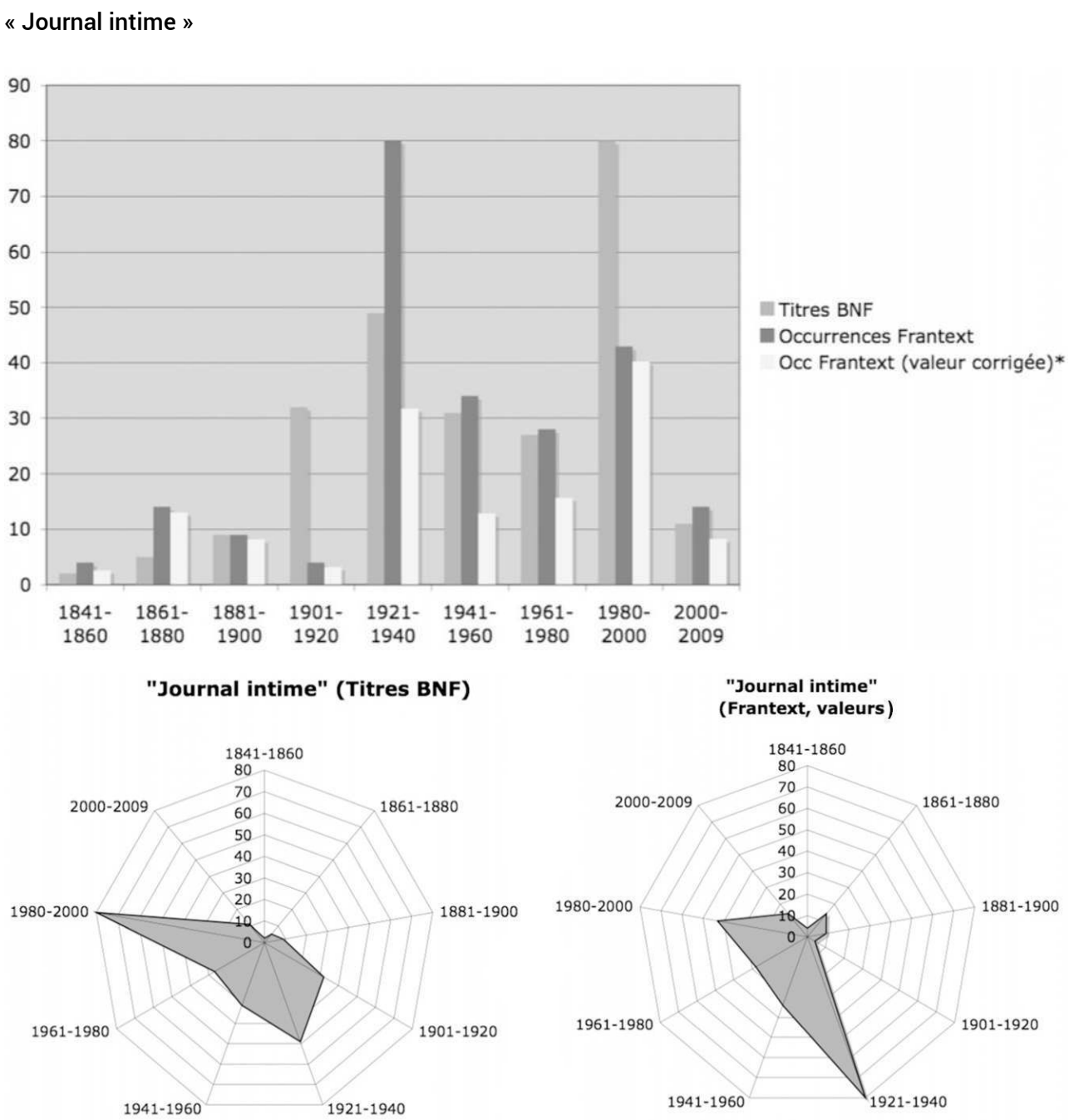

$\mathrm{Au} \mathrm{XIX}^{\mathrm{e}}$ siècle, le journal intime est l'objet d'un (logique) effet de métadiscours : ceux qui en parlent le font... dans leur journal, ou dans leur correspondance. Sur vingt-sept mentions dans Frantext pour cette période, vingt et une sont assumées par des diaristes, mémorialistes ou épistoliers : Amiel, Loti, Barrès, les Goncourt, Eugénie de Guérin. Cette dernière, qui est aussi la première, en 1840, à mentionner l'expression dans notre corpus, fait le lien entre cette activité et la pratique épistolaire, qui peut en être une sorte de variation. En avril, elle note : «Si je n'ai rien mis ici [dans son journal] depuis huit jours, c'est que je n'ai rien fait qu'écrire à Marie, écrire un journal intime, feuilles volantes d'amitié qui s'en iront joncher son lit [.... ${ }^{39}$. » Hugo rapproche également les deux genres, répondant à l'un de ses correspondants : "Votre dernière lettre nous a charmés, cher proscrit ; c'était un charmant petit journal intime qui ressemblait à votre sourire ${ }^{40}$. » Mais c'est bien sûr Amiel qui est le plus disert à propos du journal, qui semble chez lui un sujet de conversation fréquent : le 7 juin 1866, il écrit avoir "philosophé avec Philine sur les avantages et les inconvénients du journal intime ${ }^{41} »$; quelques mois plus tôt, il a soupé chez la même où " on [lui $\mathrm{a}] \mathrm{l}[\mathrm{u}]$ beaucoup de pages de journal intime ${ }^{42}$ ». Michelet, de son côté, dit avoir « causé » avec Athénaïs du «Journal intime qu'[il] a écrit de toute [leur] vie ${ }^{43}$ ». Cette vogue du journal ne manque pas d'être remarquée : Bourget, en parle déjà, dans ses Nouveaux essais de psychologie contemporaine, en 1885, comme d'une "habitude", destinée à satisfaire l'« appétit de confession ${ }^{44} »$ de ses contemporains. 
31 Le $\mathrm{xx}^{\mathrm{e}}$ siècle, en ses débuts, se nourrit de la lecture des journaux et les mentionne constamment: les occurrences de journal intime quadruplent, passant de 27 à 104 mentions dans les textes. Amiel est évidemment le champion de ces références : il est lu par Paul Bourget, qui lui consacre un chapitre entier de ses Nouveaux essais de psychologie contemporaine $^{45}$, fréquenté assidûment par Du Bos qui en parle à longueur de journal (“j'avais travaillé jusqu'à minuit et demi mon Amiel $\left.{ }^{46} »\right)$, et a par ailleurs écrit un essai sur lui, publié dans le recueil Approximations en 1921. Amiel est aussi mentionné par Gide ( « c'était le temps où le journal intime d'Amiel faisait fureur ${ }^{47}$ »), Alain-Fournier («J'ai feuilleté, oh, à peine, le journal d'Amiel $\left.{ }^{48} »\right)$, apparaît dans Silbermann, de Jacques de Lacretelle $^{49}$, dans Rêveuse bourgeoisie, de Drieu La Rochelle ${ }^{50}$, est évoqué par Thibaudet ${ }^{51}$ et Barthes $^{52}$. D'autres diaristes sont très populaires chez les écrivains, notamment Maurice et Eugénie de Guérin, mentionnés ensemble ou séparément par Barbey d'Aurevilly, les Goncourt, Amiel, Huysmans, Barrès, Bloy, Jules Renard, Alain-Fournier, Jules Romains, Mauriac, Aragon, Beauvoir, Yourcenar, et Julien Green (qui, lui, déteste la sœur ${ }^{53}$ ). Car le genre, quel que soit le succès qu'il rencontre, a ses détracteurs : nombreuses sont les remarques (souvent critiques) sur le succès de cette pratique. Jean Guéhenno dénonce le "pullulement ${ }^{54}$ » des journaux intimes, Reverdy considère qu'ils sont devenus «le confessionnal des athées ${ }^{55}$ » Green parle de "dégoût ${ }^{56}$ » à leur lecture, et l'un des personnages de Duhamel mentionne son « horreur des journaux intimes ${ }^{57}$ ». L'apparition du pluriel, qui représente plus du tiers des emplois (38 occurrences VA) est le symptôme de l'accession du journal au statut de genre; on ne l'évoque plus seulement comme une œuvre individuelle, mais comme un phénomène général, voire un matériau indistinct, pris dans la masse d'une énumération: «Le magnétophone n'est qu'une ruse nouvelle contre l'effacement, tels autrefois les journaux intimes, les Mémoires, les lettres posthumes à un lecteur-descendant ${ }^{58}$ ", " cette maison, son silence, son odeur, ses placards et ses commodes débordant de lettres, de journaux intimes, factures, coupures de presse, photos, télégrammes, devis, manuscrits ${ }^{59} »$.

L'autre indicateur littéraire, qualitatif cette fois, de la banalisation de sa pratique est le fait que le journal soit, aussi, mis en scène dans les romans: pour n'en prendre qu'un exemple (ils sont nombreux)-dans les Faux-Monnayeurs de Gide, Sarah soustrait le journal de son père pour le donner à lire à Édouard ${ }^{60}$. De plus, le journal fait œuvre et à ce titre, peut être considéré comme un modèle : «Bon-papa et bonne-maman m'ont élevé pour avoir des rentes et écrire mon journal intime comme André Gide ${ }^{61}$ ", déclare le personnage d'Yves dans Rêveuse bourgeoisie, de Drieu la Rochelle. Enfin, le sens de l'expression journal intime s'est lui aussi profondément enrichi. Françoise Simonet-Tenant soulignait «le caractère insatisfaisant d'un tel qualificatif pour caractériser ce type d'écriture ${ }^{62}$ ». On peut en effet dissocier la pratique diaristique et la profondeur des degrés d'aveu, de révélation, ainsi que la nature des événements autobiographiques évoqués. De manière symétrique, on peut, en examinant les titres des œuvres, associer journal intime à tout autre chose qu'un écrit autobiographique cadencé. Nous nous référerons, là encore, à BN-Opale Plus, qui nous livre quelques exemples de cette mutation.

$\mathrm{Au} \mathrm{Xx}^{\mathrm{e}}$ siècle, journal intime représente $31 \%$ des items de l'adjectif recensés au catalogue : il est utilisé, soit comme titre, soit comme sous-titre, dans 220 occurrences. Ce qui constitue une progression considérable relativement aux $4 \%$ (17 occurrences) du siècle précédent. Certains de ces ouvrages sont de "vrais " journaux, à savoir des écrits personnels référentiels cadencés dans le temps, et assumés par leur énonciateur. Ils 
peuvent toutefois connaitre une sorte de sectorisation, qui déplace l'accent sur le statut professionnel de l'auteur ou la période historique qu'il a vécue : La Fête des pères : journal intime d'un psychanalyste (Claude Lorin, 1995), Sensible girouette, naïvetés et jobardises d'une Française de la zone libre pendant les années 1940, extraits de son journal intime (Simone Collin, 1968). Les journaux, réels ou fictifs, sont également revendiqués comme une source d'inspiration romanesque, que l'on trouve par exemple dans les titres Coeur d'esclave. Roman extrait du journal intime d'une jeune Orientale (Paul Mado, 1931) ou dans Mademoiselle Tayrac: une vie d'institutrice d'après son journal intime, roman (Anna Sorèze, 1933). Dans certains cas, la nature fictive de ces écrits ne fait aucun doute, ne serait-ce qu'à cause de la dissociation du nom de l'auteur et de celui du (pseudo-)diariste. Dans ce cas, la mention «intime» devient une sorte de supplément sentimental, ou un apéritif publicitaire lorsqu'il s'agit d'une personnalité : Maurice Dekobra, auteur prolifique à succès de la première moitié du $\mathrm{xx}^{\mathrm{e}}$ siècle ${ }^{63}$, propose ainsi en 1951 Cafard mauve, journal intime d'une femme de 49 ans, tandis que plus près de nous Christine Clerc signe un ouvrage intitulé Journal intime de Jacques Chirac. Enfin, les auteurs se servent parfois de l'adjectif, en jouant sur le sème de l'érotisme, pour pimenter le titre (Mes amants de vacances, journal intime d'une femme libre, 1984). L'un des détournements les plus flagrants du genre a sans doute été le Journal intime de Sally Mara, rédigé par Raymond Queneau et publié aux Éditions du Scorpion en 1935. L'auteur de Zazie (qui était lui-même diariste) a utilisé cette forme pour mettre en scène les confessions fictives d'une jeune Irlandaise, dont la prétendue naïveté est le prétexte à d'incessantes allusions à la sexualité. Le burlesque est si présent, et les sous-entendus si appuyés, qu'il est impossible de se tromper quant à la nature fictionnelle du texte.

Cet essor multidirectionnel des usages d'intime place le mot, d'un point de vue pragmatique, dans une situation relativement illogique, et consacre le paradoxe qui a été notre point de départ: le caractère d'intimité est désormais utilisé pour assurer une publicité maximale à ce qui est supposé se restreindre, électivement, à une communication privée. L'on ignore encore si cette inflation, constatable en ce début de xxI ${ }^{e}$ siècle (qui compte déjà 94 titres comprenant «journal intime » en huit ans - contre 220 pour la totalité du $\mathrm{xx}^{\mathrm{e}}$ siècle !) sera une tendance durable, ou un simple effet de mode générationnel. Mais il est évident que ni la nature intime ni le caractère autobiographique des écrits ne sont plus garantis par cette appellation, désormais investie par le fictionnel. Comme le note Thomas Clerc, il convient désormais de « distinguer le genre de son nom ${ }^{64}$ ».

Plutôt qu'à une jungle, l'intime, cette "notion éminemment labile ${ }^{65}$ » mériterait sans doute d'être comparé à un jardin anglais: il en a les sinuosités, les subtilités, la variété chatoyante. Ses acceptions, comme les allées, suivent des courbes sémiques fluides, qui l'ont entraîné de l'intériorité extrême (le fameux intimus) de l'étymologie à une publicité assumée et paradoxale. Les variations de définitions lexicographiques, leurs nuances, sont les miroirs des mutations du corps social lui-même, promoteur, à partir de la fin du XVIII siècle, d'un intime qui ne soit plus seulement un mode relationnel, mais une conquête de l'intériorité. Mais le cheminement sémantique d'intime, sous ses allures souples, se fait d'une manière très organisée: on peut repérer un premier déplacement latéral, qui recentre l'intimité du relationnel vers l'individu, puis un mouvement d'expansion, qui accroît le territoire de l'intime et le fixe comme un périmètre protégé, mais partageable. La cartographie statistique des titres d'ouvrages et des textes nous permet de prendre la mesure de cette extension; elle nous raconte la fortune du mot, appliqué à des objets 
nombreux et divers, puis essentialisé, et nous laisse deviner les pratiques nouvelles dont la notion est devenue le centre, au fil du "bougé» des significations : confidences, conversation, entretiens, écrits. À partir du $\mathrm{xIX}^{\mathrm{e}}$ siècle, l'intime connaît un lieu d'expression privilégié : le journal. Les co-occurrents, indicateurs parcellaires, mais précis, nous montrent comment la langue se cristallise autour de ce syntagme, qui finit presque par se lexicaliser, alors que sa nature, elle, change, et que l'intimus dont il se charge devient aussi un extime de plus en plus souvent livré au champ éditorial.

Victime de son succès, en quelque sorte, le mot intime est aujourd'hui la proie d'une entropie qui est en train de le vider de son sens : car pour la première fois dans son histoire, on l'utilise, en contexte, pour lui faire dire le contraire de ce qu'il est supposé signifier. Ce ne sont pas selon nous les usages linguistiques (les contradictions génériques relevées dans les titres, par exemple) qui sont les artisans de cette démonétisation sémique, mais le fait que ces étiquettes relèvent, de manière flagrante, de stratégies éditoriales et publicitaires: d'où une défiance, voire une répugnance, envers ces écrits « intimes » qui n'en sont pas. La représentation même que nous avons de l'intimité a elle aussi profondément changé, sous l'effet des médias de grande consommation entre autres : concept perdu, flouté, floué, déplacé, exposé aux caméras de télévision, disséminé sur les blogs, l'intime s'est fragilisé, ce qui explique peut-être la soif de théorisation dont il fait l'objet, et que l'on pourrait lire comme une tentative pour fixer une réalité - fût-elle immatérielle - que l'on perçoit comme menacée. Mais, les mots (comme les tabous) ont parfois la vie dure : la riche histoire lexicographique d'intime doit aussi nous rassurer sur la plasticité du terme, et sa capacité à agréger des acceptions relativement éloignées de son étymologie. Certes, ce qu'il désigne a changé de contour, et le dévoilement de la vie personnelle, à plus ou moins grande échelle, est aujourd'hui tenu comme une sorte de passage obligé du partage social. Cela dit, l'idéal de transparence contemporain, dont le mythe est entretenu par des communautés médiatiques Internet, n'est peut-être que la surface d'une nouvelle intériorité. Dans un paysage saturé par les impératifs catégoriques de discours constamment injonctifs (comment manger, aimer, consommer, croire...), l'intime continue à former un espace de résistance, de conviction ou de trouble, de rêve ou d'abandon, de ferveur et de solitude : un endroit, finalement, où être soi.

\section{NOTES}

1. Élisabeth Lebovici, "L'intime et ses représentations ", dans Élisabeth Lebovici (dir.), L'Intime [1998], Paris, École Nationale Supérieure des Beaux-Arts, 2004, p. 20.

2. Maine de Biran, Journal, t. I [1824], Neufchatel, La Baconnière, 1955, p. 426.

3. Nathalie Sarraute, Enfance, Paris, Gallimard, 1983, p. 266.

4. Jean-Pierre Dufief, Les Écritures de l'intime de 1800 à 1914, Rosny, Bréal, 2001, p. 110.

5. Philippe Ariès et Georges Duby (dir.), Histoire de la vie privée, t. V, Paris, Seuil, coll. "Points », 1999, p. 64.

6. Françoise Simonet-Tenant, Le Journal intime. Genre et écriture ordinaire, Paris, Téraèdre, 2004, p. 13. 
7. L'auteur remercie Annie Bernardoff, du centre de documentation de l'ATILF-CNRS, pour son aide aussi amicale que précieuse dans la mise à disposition des dictionnaires anciens et des dossiers de mots (archives du TLF).

8. Nous soulignons.

9. Jean Beauverd, "Problématique de l'intime », dans Intime, intimité, intimisme, Lille, Université de Lille III, Éditions Universitaires, 1976, p. 16.

10. Nous soulignons.

11. Pour rendre les scores lisibles, nous avons exprimé une valeur absolue ou VA (le nombre d'occurrences relevé dans le corpus pour chaque période concernée), et une valeur relative (ou VR), pondérée par le nombre de textes effectivement présents dans la base pour chacune des tranches temporelles en question.

12. Alain Girard, "Évolution sociale et naissance de l'intime», dans Raphaël Molho et Pierre Reboul (dir.), op. cit., p. 53.

13. Annick Pardailhan-Galabrun (dir.), La Naissance de l'intime, Paris, PUF, coll. « Histoires », 1988.

14. Cet outil, édité par Micro-Application, nous propose des «interfaces graphiques colorées adaptées à tous les styles (adolescents ou adultes) » et une "alerte rouge " pour faire fuir les indiscrets.

15. Marie Chaix, L'Âge du tendre, Paris, Seuil, 1979, p. 39.

16. Violette Leduc, La Bâtarde, Paris, Gallimard, 1964, p. 214.

17. Gabriel Matzneff, Ivre du vin perdu [1981], Paris, Gallimard, coll. « Folio », 1992, p. 19.

18. Edmond et Jules de Goncourt, Journal : mémoires de la vie littéraire, t. III [1890], Paris, Fasquelle et Flammarion, 1959, p. 370.

19. Paul Bourget, Le Disciple [1889], Paris, Plon, 1901, p. 141.

20. Léon Bloy, Le Mendiant ingrat : Journal 1892-1895 [1894], Paris, Mercure de France, 1956, p. 100.

21. Maurice Barrès, Mes cahiers [1907], Paris, Plon, 1932, p. 146.

22. Jacques Maritain, Humanisme intégral : problèmes temporels et spirituels d'une nouvelle chrétienté, Paris, Aubier, 1939, p. 308.

23. Pierre Reverdy, Le Livre de mon bord. Notes 1930-1936, Paris, Mercure de France, 1948, p. 37.

24. Paul Valéry, Variété V, Paris, Gallimard, 1948, p. 109.

25. André Gide, Ainsi soit-il ou les Jeux sont faits, dans Journal 1939-1949, Paris, Gallimard, coll. «Bibliothèque de la Pléiade », 1960, p. 1186.

26. Marcel Proust, Le Temps retrouvé [1922], Paris, Gallimard, coll. « Bibliothèque de la Pléiade », p. 715.

27. Saint-John Perse, Éloges [1911], Paris, Gallimard, 1986, p. 41.

28. Louis Aragon, Le Roman inachevé, Paris, Gallimard, 1956, p. 235.

29. Gabriel Matzneff, op. cit., p. 238.

30. Jean-François Sablayrolles, La Néologie en français contemporain, Paris, Champion, 2000, p. 177 : «Le comportement syntaxique d'une lexie fait partie intégrante de sa définition et tout changement relève de la néologie. »

31. Les trois titres employant "l'intime » renvoient eux à une substantivation bien antérieure, attestée dès 1752 (Trévoux), et qui désigne le ou la proche amie : L'Intime ou lequel des deux (drame en trois actes d'Alfred Mouron, 1836). Plus ambigus sont les deux cas de Boquillon ou l'Intime du vieux mari (une comédie de Louis Boquillon, 1989) et de L'Intime. Poésies, de Michel Dolques (1899). 32. Philippe Lejeune, "Cher cahier...». Témoignages sur le journal personnel, Gallimard, coll. « Témoins », 1989, p. 12.

33. Comme cela a été précisé dans le "Préambule», on en trouve un emploi beaucoup plus ancien, cependant, dans Thibaudet: «C'est, comme journal de la France, tout ce qu'on peut imaginer de plus extime. » (Réflexions sur la littérature [1936], Paris, Gallimard, 1938, p. 223).

34. Françoise Simonet-Tenant, op. cit., p. 18. 
35. Philippe Lejeune, Le Moi des demoiselles. Enquête sur le journal de jeune fille, Paris, Seuil, 1993, p. 17 : «Disons en gros que c'est en 1850 que la pratique semble s'établir. Je ne connais aucun autre exemple dans la première moitié du siècle. Cela ne veut pas dire que ça n'existait pas: simplement, on ne l'a pas conservé. »

36. Pierre Pachet, Les Baromètres de l'âme, Paris, Pluriel, 2001, p. 175.

37. Béatrice Didier, Le Journal intime, Paris, PUF, 1976, p. 31.

38. Voir Thomas Clerc, Les Écrits personnels, Hachette, coll. « Ancrages », 2001, p. 66.

39. Eugénie de Guérin, Journal et Lettres, Paris, Didier, 1863, p. 371.

40. Victor Hugo, Correspondance [1849-1866], t. II, Paris, Albin Michel, 1950, p. 172.

41. Henri Frédéric Amiel, Journal intime de l'année 1866, Paris, Gallimard, 1959, p. 313.

42. Ibid., p. 119.

43. Jules Michelet, Journal, t. II : 1849-1860, Paris, Gallimard, 1962, p. 355.

44. Paul Bourget, Nouveaux essais de psychologie contemporaine [1885], Paris, Alphonse Lemerre, 1886.

45. Paul Bourget, op. cit., p. 253-304.

46. Charles du Bos, Journal t. I (1921-1923), Paris, Correa, 1946-1950, p. 16.

47. André Gide, Si le grain ne meurt, Paris, Éditions de «La Nouvelle Revue française », 1924, p. 481.

48. Alain-Fournier, Correspondance avec Jacques Rivière (1905-1914) [1914], Paris, Gallimard, 1930, p. 175.

49. Jacques de Lacretelle, Silbermann [1922], Paris, Gallimard, 1933, p. 77 : « Apercevant un livre posé sur la table à ouvrage de ma mère, [il] le retourna pour en voir le titre. C'était, il m'en souvient, le journal intime d'Amiel. »

50. Pierre Drieu la Rochelle, Rêveuse bourgeoise [1937], Paris, Gallimard, 1960, p. 289.

51. Albert Thibaudet, op. cit., p. 223.

52. Roland Barthes, Nouveaux essais critiques, Paris, Seuil, coll. « Points », 1972, p. 165.

53. Julien Green, Journal, t. IV : 1943-1946, Paris, Plon, 1949, p. 146 : «Quelle insipidité ! Elle a l'esprit tellement banal qu'elle atteint parfois à une sorte de profondeur. »

54. Jean Guéhenno, Jean-Jacques, t. III. Grandeur et Misère d'un esprit : 1758-1778, Paris, Gallimard, 1952, p. 164.

55. Pierre Reverdy, Le Livre de mon bord [1936], Paris, Mercure de France, 1948, p. 32.

56. Julien Green, Journal, t. IV (1943-1946), Paris, Plon, 1949, p. 191.

57. Georges Duhamel, Chronique des Pasquier I. Le notaire du Havre [1933], Paris, Mercure de France, 1948 , p. 25.

58. Jacques Roubaud, Le Grand Incendie de Londres, Paris, Seuil, 1989, p. 97.

59. Michel Tournier, Le Vent Paraclet [1977], Paris, Gallimard, coll. « Folio », 1993, p. 268.

60. André Gide, Les Faux-monnayeurs, dans Romans, Paris, Gallimard, coll. «Bibliothèque de la Pléiade ", 1958, p. 1019.

61. Drieu La Rochelle, Rêveuse bourgeoise, Paris, Gallimard, 1937, p. 289.

62. Françoise Simonet-Tenant, Le Journal intime. Genre littéraire et écriture ordinaire, Paris, Téraèdre, 2004, p. 18.

63. Il a écrit plus d'une trentaine de romans, dont La Madone des Sleepings, roman cosmopolite, Paris, Baudinière, 1925.

64. Thomas Clerc, op. cit., p. 63.

65. Françoise Simonet-Tenant, «À la recherche des prémices d'une culture de l'intime », infra. 


\section{RÉSUMÉS}

Depuis sa première apparition dans les dictionnaires français du XVII ${ }^{\mathrm{e}}$ siècle, l'acception du terme intime s'est diversifiée et étendue : comme adjectif qualificatif réservé à une relation d'affection ou d'amitié, il s'utilisait alors dans plusieurs domaines (la théologie, les sciences naturelles, la psychologie) et il a finalement obtenu le statut de substantif à la fin du $\mathrm{xx}^{\mathrm{e}}$ siècle. L'analyse de son évolution lexicographique a été ici comparée à ses usages dans la langue des textes littéraires et des titres d'œuvres publiées en France entre 1600 et 2009. L'interprétation de ses cooccurrents a mené à l'apparition d'une tendance du même nom à la fin du XIX siècle et à l'application du terme à divers objets qui, paradoxalement, ont aujourd'hui introduit la notion d' intime dans la sphère publique.

Since it first appeared in the French dictionaries of the seventeenth century, the acceptance of the term intime has diversified and proliferated: as a qualifier reserved for a relationship of affection or friendship, it was then used in a number of fields (theology, the natural sciences, psychology) and finally achieved the status of substantive at the end of the twentieth century. The analysis of its lexicographie evolution has been compared here to its uses in language in the literary texts and the titles of works published in France between 1600 and 2009. The interpretation of its co-occurrents led to the appearance of a trend of the same name at the end of nineteenth century, and to the application of the term to various objects, which, paradoxically, introduced the notion of intimate into the public sphere today.

\section{INDEX}

Mots-clés : intime, journal intime, lexicométrie, Frantext, catalogue général de la BNF

Keywords : intimate, diary, lexicometry, Frantext, French national library catalogue

\section{AUTEUR}

\section{VÉRONIQUE MONTÉMONT}

Université Henri-Poincaré de Nancy, ATILF, IUF 